\title{
Grape skin extracts from winemaking by-products as a source of trapping agents for reactive carbonyl species
}

\section{Pedapati SC Sri Harsha, ${ }^{a}$ Marta Mesias, ${ }^{b}$ Vera Lavellia and Francisco J Morales ${ }^{b}$}

${ }^{a}$ DeFENS, Department of Food, Environmental and Nutritional Sciences, Università degli Studi di Milano, 20133 Milan, Italy

'Institute of Food Science, Technology and Nutrition, ICTAN-CSIC, Madrid 28040, Spain

\begin{abstract}
BACKGROUND: Clinical evidence supports the relationship between carbonyl stress and type II diabetes and its related pathologies. Methylglyoxal (MGO) is the major dicarbonyl compound involved in carbonyl stress. Efforts are therefore being made to find dietary compounds from natural sources that could exert an MGO trapping response.

RESULTS: The in vitro MGO trapping capacity of six red and seven white grape skin extracts (GSE) obtained from winemaking by-products was investigated. Methanolic GSE exhibited a promising MGO trapping capacity that was higher in red GSE (IC50 $2.8 \mathrm{mg} \mathrm{mL}^{-1}$ ) when compared with white GSE (IC50 $3.2 \mathrm{mg}$ $\mathrm{mL}^{-1}$ ). The trapping ability for red GSE correlated significantly with total phenolic content and antioxidant capacity. However, no correlations were observed for white GSE, which suggests that other compounds were involved in the trapping activity.

CONCLUSION: GSE may be considered a natural source of carbonyl stress inhibitors, thus opening up its possible utilization as a nutraceutical ingredient. Further investigations are required to understand the mechanism involved in the carbonyl trapping ability of red and white grape skin samples and their relationship with glycation.
\end{abstract}

KEYWORDS: trapping; methylglyoxal; dicarbonyl compounds; antiglycation; grape skin

\section{INTRODUCTION}

The global food industry is facing an uphill task with respect to the utilization of its by-products.' Many epidemiological studies have reported that consumption of phytochemical-rich fruits and vegetables significantly reduces the incidence of several chronic diseases. ${ }^{2-4}$ There is therefore a need to develop new applications involving the use of by-products from the fruit and vegetable industry that could provide a source of phytochemicals offering promising health benefits.

Owing to changing lifestyles and nutritional habits, there has been an upsurge in the prevalence of diabetes, with an estimated 300 million possible registered cases worldwide by $2030 .{ }^{5}$ Previous confirmatory reports suggest that hyperglycemia complications play a major part in the onset and progression of type II diabetes. Interestingly, there is an overwhelming body of evidence indicating that the two major $\alpha$-dicarbonyl reactive species, i.e. glyoxal and methylglyoxal (MGO), cause carbonyl stress, which is one of the root causes of hyperglycemia in diabetes. MGO is an important precursor of the end products formed during the process of protein glycation i.e. advanced glycation end products (AGEs). ${ }^{6}$ Dicarbonyl compounds can be formed either from sugars or amino-carbonyl reaction intermediates or during glycolysis under in vivo conditions. ${ }^{7}$ A two- to six fold increase in MGO in the plasma of diabetic patients can be observed when compared with healthy individuals. ${ }^{8}$ An increase in MGO levels leads to a decrease in glutathione, which in turn leads to adverse changes in the antioxidant defense system and ultimately affects metabolic routes. ${ }^{9}$ Moreover, 
reactions between $\alpha$-dicarbonyl compounds and proteins occur at a much faster rate than with sugars, bringing about the formation of protein adducts in the glycation process. ${ }^{10}$

Several synthetic organic compounds such as aminoguanidine, metformin and pyridoxamine have been shown to have excellent carbonyl trapping capacities." Since there are increasing concerns about the side effects of these compounds, efforts are being made to find dietary compounds fromnatural sourceswhich could exert similar trapping responses. Phenolic compounds present in these sources may not only act as metal chelators and radical scavengers, but also as scavengers of radical carbonyl species. ${ }^{12}$ It is well known that berries and grapes are rich in flavonoids and have demonstrated their ability to inhibit protein glycation ${ }^{13}$ and scavenge reactive carbonyls. ${ }^{14}$ Certain flavonoids such as luteolin, quercetin and rutin have also been shown to be more efficient inhibitors of AGEs in the early stage of protein glycation than aminoguanidine. ${ }^{15}$

Previous research found that grape skin extracts (GSE) obtained from winemaking by-products were effective sources of antiglycation agents. ${ }^{16,17}$ The antiglycative activity of these extracts was similar to that of phenolic-rich herbs such as cinnamon, rosemary and sage. ${ }^{18-20}$ Antiglycative activity is commonly related to the antioxidant potential of the natural extracts, as several flavonoids' structural requirements for AGE inhibitory activity were found to be similar to those of radical scavenging activities. ${ }^{12}$ As the inhibition of AGE formation can be carried out at different stages of the glycation and sugar autooxidation, ${ }^{21}$ it would seem that the scavenging of reactive dicarbonyls is a plausible mechanism. The aim of the present study was focused on the ability of GSE to trap reactive carbonyl species and potentially explain their antiglycative properties. For this purpose, phenolic-rich methanolic extracts of red and white grape skins were tested for their dicarbonyl trapping abilities using an in vitro model system under simulated physiological conditions $\left(\mathrm{pH} 7.4,37^{\circ} \mathrm{C}\right)$.

\section{MATERIALS AND METHODS}

\section{Chemicals}

Gallic acid, Trolox (6-hydroxy-2,5,7,8-tetramethylchroman-2-carboxylic acid), 2,2 ' -azinobis(3ethylbenzothiazoline-6-sulfonic acid) diammonium salt (ABTS), methylglyoxal (MGO), 5-methylquinoxaline (5-MQ) and o-phenyldiamine (OPD) were purchased from Sigma (St Louis, MO, USA). Folin-Ciocalteu reagent, iron(III) chloride, potassium persulfate and sodium bicarbonate were obtained from Panreac Quimica (Barcelona, Spain). Sodium dihydrogen phosphate monohydrate, sodium phosphate dibasic dodecahydrate, glacial acetic acid and high-performance liquid chromatography (HPLC)-grade methanol were acquired from Merck (Darmstadt, Germany). 2,4,6-Tri(2-pyridyl)-striazine (TPTZ) and pyridoxamine (PM) were purchased from Fluka Chemical (Madrid, Spain). The Milli-Q water used was produced using an Elix3 water purification system coupled to an Advantage I0 Milli-Q module (Millipore, Molsheim, France). All other chemicals and reagents were of analytical grade.

\section{Collection of grape pomace}

Procurement of grape pomace varieties, milling and sieving procedures were as previously described by Sri Harsha et al. ${ }^{16}$ Barbera (BA), Dolcetto (DO), Croatina (CR), Freisa (FR), Neretto (NR), and Grignolino (GR) were selected as red grape skin varieties, while Chardonnay $(\mathrm{CH})$, Muller Thurgau (MT), Arneis (AR), Erbaluce (ER), Nascetta (NA), Moscato (MO), and Riesling (RI) were selected as white grape skin varieties. Samples were kindly provided by different winemakers in northern Italy. The pomace samples were sieved 
with a $5 \mathrm{~mm}$ sieve at the wineries to separate the skins from the seeds and were transported frozen to the lab. The samples were dried at $50^{\circ} \mathrm{C}$ for $8 \mathrm{~h}$, milled, sieved to obtain fractions having particle sizes in the range $125-250 \mu \mathrm{m}$, and stored in the dark at $4^{\circ} \mathrm{C}$ until analysis.

\section{Preparation of grape skin extract}

Grape skin powder $(\sim \mathrm{Ig})$ was continuously extracted for $2 \mathrm{~h}$ at room temperature added with $16 \mathrm{~mL}$ methanol-water- $\mathrm{HCl}(80: 20: 0.1, \mathrm{v} / \mathrm{v} / \mathrm{v})$ solution for red grape skins, and $16 \mathrm{~mL}$ methanol-water $(80: 20, \mathrm{v} / \mathrm{v})$ solution for white grape skins. $\mathrm{HCl}$ was used for extraction of red grape skins to enhance stability of anthocyanins as described by Sri Harsha et al. ${ }^{16}$ The mixture was centrifuged at $5000 \mathrm{rpm}$ for $10 \mathrm{~min}$, the supernatant was recovered and the solid residue was resuspended with $12 \mathrm{~mL}$ of the same solvent twice, repeating the same procedure. The supernatants were pooled and the volume was reduced using a rotary evaporator (Strike 300 Steroglass, Perugia, Italy) and made up to $10 \mathrm{~mL}$ with the same solvent. The extract thus obtained was stored at $-20^{\circ} \mathrm{C}$ until further analysis.

\section{In vitro evaluation of direct MGO trapping capacity}

Direct MGO trapping capacity was performed as described by Peng et al., ${ }^{22}$ with some modifications. ${ }^{23}$ Pyridoxamine (PM) was used as the positive control. A $100 \mu \mathrm{L}$ aliquot of MGO solution was mixed with 750 $\mu \mathrm{L}$ phosphate-buffered saline (PBS), $50 \mu \mathrm{L} 5$-MQ (used as an internal standard) and $100 \mu \mathrm{L}$ of either PBS (blank), extract solutions or PM solution. The final concentration of each reactant in the reaction medium was $0.04 \mathrm{mgmL}^{-1}$ for $\mathrm{MGO}, 0.05 \mathrm{mgmL}^{-1}$ for $5-\mathrm{MQ}$, a range of $0.00 \mathrm{I}-0.1 \mathrm{mgmL}^{-1}$ for $\mathrm{PM}$ and a range of 0 $10 \mathrm{mgmL}^{-1}$ for GSE (being up to $5 \mathrm{mgmL}^{-1}$ for red GSE and up to $10 \mathrm{mgmL}^{-1}$ for white GSE). Blank, positive control and samples were incubated at $37^{\circ} \mathrm{C}$ for $168 \mathrm{~h}$ in an oven (Memmert, Wuppertal, Germany). After the incubation period, $200 \mu \mathrm{L}$ OPD were added (final concentration: $1.8 \mathrm{mgmL}^{-1}$ ). The unreacted MGO was quantified by HPLC (Shimadzu, Kyoto, Japan) on the basis of the amount of the derivatized product, 2-methylquinoxaline (2-MQ). The chromatographic separation was carried out on a Mediterranean Sea ODS2 column (I50 × $3 \mathrm{~mm}, 5 \mu \mathrm{m}$, Tecknokroma, Barcelona, Spain). Percentage decrease in MGO trapping capacity at $168 \mathrm{~h}$ was calculated using the following equation: MGO decrease $\%=$ [(amount of MGO in blank - amount of MGO in sample or PM solution) / amount of MGO in blank] $\times$ $100 \%$. The IC50 values of samples were calculated from the dose-response curves using Microsoft Excel computer software. The $\mathrm{IC}_{50}$ value $\left(\mathrm{mg} \mathrm{mL}^{-1}\right)$ represents the dosage of extract necessary to trap MGO by $50 \%$.

\section{Kinetics of MGO trapping}

Based on the $I C_{50}$ values of the samples for MGO trapping capacity, a suitable concentration of $2.5 \mathrm{mgmL}^{-1}$ for red GSE and $5 \mathrm{mgmL}^{-1}$ for white GSE were selected for the kinetic evaluation of the MGO trapping capacity. The reaction mixture contained a $100 \mu \mathrm{L}$ aliquot of MGO solution mixed with $750 \mu \mathrm{L}$ PBS, $50 \mu \mathrm{L}$ $5-\mathrm{MQ}$ and $100 \mu \mathrm{L}$ PBS (blank), GSE solutions or PM solution. MGO trapping capacity was studied at different time intervals $(0,24,48,72,96$ and $168 \mathrm{~h})$. Unreacted MGO was determined by HPLC analysis as described previously. Comparison of the reactivity between samples at the same dosage was assessed by calculating the time (h) necessary to trap MGO by $50 \%$.

\section{Determination of total phenolic content}

Total phenolic content (TPC) in the extracts was determined by the Folin-Ciocalteu method as described by Singleton et al. ${ }^{24}$ and adapted to a plate reader as described by Mesías et al. ${ }^{23} \mathrm{In}$ a $10 \mathrm{~mL}$ tube, $100 \mu \mathrm{L}$ of the GSE sample (appropriately diluted if necessary) and $250 \mu \mathrm{L}$ Folin-Ciocalteu reagent (diluted I:I (v/v) in 
methanol) were mixed and vortexed. After exactly $3 \mathrm{~min}, 500 \mu \mathrm{L}$ of $75 \mathrm{~g} \mathrm{~L}^{-1}$ sodium carbonate solution and $4 \mathrm{~mL}$ methanol-water (50:50, v/v) were added; then the mixture was vortexed for a further $10 \mathrm{~min}$ and allowed to stand at room temperature in darkness for $60 \mathrm{~min}$. The absorbance at $750 \mathrm{~nm}$ was recorded using a Synergy TM HT-multimode microplate spectrophotometer (BioTek Instruments, Winooski, VT, USA) and quantified using gallic acid as a standard. BioTek Gen5TM data analysis software was used. Results were expressed as $\mathrm{mg}$ gallic acid equivalent (GAE) $\mathrm{g}^{-1}$ sample. Limit of quantification was set at $1.9 \mathrm{mg}$ GAE $\mathrm{g}^{-1}$ sample.

\section{FRAP assay}

Ferric reducing antioxidant power (FRAP) was determined as described by Morales et al. ${ }^{25}$ Absorbance reading was taken using a microplate reader as described above. Aqueous solutions of Trolox at various concentrations were used for calibration. All measurements were performed in triplicate. Results were expressed as $\mu \mathrm{mol}$ Trolox equivalent antioxidant capacity (TEAC) $\mathrm{g}^{-1}$ sample. Limit of quantitation was set at $3.8 \mu \mathrm{mol}$ TEAC $\mathrm{g}^{-1}$ sample.

\section{ABTS assay}

Antioxidant activity was estimated in terms of the radical scavenging activity of samples following the procedure described by Delgado-Andrade and Morales $^{26}$ and modified by Mesías et al. ${ }^{23}$ ABTS cations (ABTS -+) were produced by reacting $7 \mathrm{mmol} \mathrm{L}^{-1} \mathrm{ABTS}$ stock solution with $2.45 \mathrm{mmol} \mathrm{L^{-1 }}$ potassium persulfate and allowing the mixture to stand in the dark at room temperature for $12-16 \mathrm{~h}$ before use. The ABTS-+ solution was diluted with distilled water to an absorbance of $0.70 \pm 0.02$ at $734 \mathrm{~nm}$. $40 \mu \mathrm{L}$ of sample (appropriately diluted if necessary) was added to $200 \mu \mathrm{L}$ water and $40 \mu \mathrm{L}$ diluted ABTS•+ solution and an absorbance reading was taken after $10 \mathrm{~min}$ using a microplate reader as described above. Aqueous solutions of Trolox at concentrations of $0.016-0.5 \mathrm{mmol} \mathrm{L}^{-1}$ were used for calibration. Limit of quantification was set at I.I $\mu \mathrm{mol}$ TEAC $\mathrm{g}^{-1}$ sample.

\section{Statistical analysis}

Statistical analyses were performed using Statgraphics Centurion XV (Herndon, VA, USA). Data were expressed as mean \pm standard deviation (SD). Analysis of variance (ANOVA) and least significant difference (LSD) test were applied to determine differences between means. Differences were considered to be significant at $\mathrm{P}<0.05$. Relationships between the different parameters analyzed were evaluated by computing Pearson linear correlation coefficients at the $\mathrm{P}<0.05$ confidence level.

\section{RESULTS}

Reactive carbonyl species, such as MGO, are critical intermediates formed during glycation of proteins in vivo and from food processing. Clinical investigations have already clearly shown the relationship between MGO and type II diabetes and its related pathologies." Hence trapping reactive carbonyl species could be one of the promising alternatives to ameliorate the physiological consequences of glycation. This investigation explores the use of selected GSE as effective quenchers of MGO under simulated physiological conditions.

Screening was carried out to classify the MGO trapping activity for different red and white GSE and the results are shown for red and white GSE in Fig. I (A,B, respectively). Due to the different reactivity observed among varieties, concentrations ranged from 0 to $5 \mathrm{mgmL}^{-1}(\mathrm{n}=6)$ for red GSE samples (Fig. IA) and from 0 to $10 \mathrm{mgmL}^{-1}(\mathrm{n}=7)$ for white GSE samples (Fig. IB). Five groups of samples (R-GI, R-G2, R-G3, 
R-G4, and R-G5) were identified for red GSE. CR and DO varieties did not show significant differences and therefore they were placed in group R-G2. In a similar way, white GSE samples were divided into three groups, these being CH, MO (W-GI); MT, AR, NA (W-G2); and ER, RI (W-G3). The grouping was done based on the trapping capacities of the extracts, where samples showing similar activities were represented with the same trend line (mean $\pm S D$ ). The MGO trapping assay was highly reproducible and the standard deviation for each sample was lower than $2 \%$.

Red GSE efficiently trapped $\sim 80-90 \%$ of MGO at $5 \mathrm{mgmL}^{-1}$ concentration except for GR and NR extracts (Fig. IA). As white GSE samples showed lower trapping activity when compared with red GSE, it was necessary to increase the concentration to $10.0 \mathrm{mgmL}^{-1}$ (Fig. IB) in order to calculate the maximum trapping activity and IC50 values. A wide range of variation in MGO trapping activity was observed among red GSE. In contrast, the variability was lower for white GSE samples. This fact can be evidenced in the $I_{50}$ values, where the concentration required to trap $50 \%$ of the MGO present in the reaction medium was estimated for each of the extracts. For red GSE, the concentrations for IC50 ranged from 1.0 to $6.5 \mathrm{mgmL}-$ I, with a mean of $2.8 \pm 2.0 \mathrm{mgmL}^{-1}$ (Fig. 2). The maximum IC50 represented an even outlier value in the box-plot representation, which corroborates the high variability shown by these extracts. The FR extract had the lowest $I C_{5} 0$, associated with its high MGO trapping capacity. In contrast, the NR extract showed the highest $\mathrm{IC}_{50}$, corresponding to its low MGO trapping capacity. In the case of NR extracts, concentrations of 7.5 and $10.0 \mathrm{mgmL}^{-1}$ were additionally tested in order to calculate their $\mathrm{IC}_{50}$ values, since at $5.0 \mathrm{mgmL}^{-1}$ the MGO trapping capacity reached only $38 \%$. All the extracts exhibited $I C_{50}$ values about 50 -fold higher than that of pyridoxamine $\left(0.05 \mathrm{mgmL}^{-1}\right)$. For white GSE, the concentrations for $I_{50}$ ranged from 1.8 to 4.5 $\mathrm{mgmL}^{-1}$ (range: $2.7 \mathrm{mgmL}^{-1}$ ), with a mean of $3.2 \pm 1.0 \mathrm{mgmL}^{-1}$. This range was within the broad range shown for red GSE and, therefore, no significant differences were observed in the $\mathrm{IC}_{50}$ mean in the two types of grapes. $\mathrm{CH}$ (included in $\mathrm{W}-\mathrm{GI})$ had the lowest $\mathrm{IC}_{50}$ value $\left(1.8 \mathrm{mgmL}^{-1}\right)$, whereas $\mathrm{RI}$ (included in WG3) had the highest $I C_{50}$ value $\left(4.5 \mathrm{mgmL}^{-}\right)$. White GSE also had $I C_{50}$ values higher than that of pyridoxamine.

Based on the former results, a more detailed kinetic study was performed in order to evaluate the trapping efficiency for each extract throughout the $168 \mathrm{~h}$ experiment at $24 \mathrm{~h}$ intervals. To carry out this kinetic monitoring, the $\mathrm{IC}_{50}$ values for the MGO trapping activity were considered and concentrations of 2.5 and 5.0 $\mathrm{mgmL}^{-1}$ were selected for red and white GSE samples, respectively. These concentrations were chosen since most of the extracts surpassed $50 \%$ of MGO trapping without reaching their maximum activity (Fig. 3). The results obtained from this kinetic study revealed that FR (R-GI) had the highest MGO trapping efficiency among the red GSE, scavenging $50 \%$ of the carbonyl compound in only $18 \mathrm{~h}$, which was lower than the time required for PM at the tested concentration ( $20 \mathrm{~h}$ ) (data not shown). BA (R-G3) needed II $4 \mathrm{~h}$ to trap half of the MGO in the reaction medium, whereas GR (R-G4) and NR (R-G5) did not reach $50 \%$ of MGO trapping during the $168 \mathrm{~h}$ experiment, as was expected according to the trend of these samples in the previous trapping activity assays (Fig. 3A). The higher variability between the red GSE when compared with white GSE is again evident in the kinetic experiment (Fig. 3). Regarding white GSE, all the extracts trapped $50 \%$ of the MGO during the $168 \mathrm{~h}$ experiment at the tested concentrations, ranging from $30 \mathrm{~h}$ (MO included in W-GI) to II $4 \mathrm{~h}$ (ER, included in W-G3), which indicates inversely the MGO trapping efficiency for the samples (Fig. 3B).

Regardless of the levels reached for their trapping activities, it should be emphasized that all of the extracts surpassed $\sim 40-50 \%$ of their final trapping activity within the first $24 \mathrm{~h}$ of the kinetic study, which explains 
the efficiency of the extracts to trap reactive carbonyl species in such a short span of time. During this short time span, FR (R-GI) and MT (included in W-G2) extracts even reached $66 \%$ and $62 \%$ of their maximum activity, respectively.

Characterization of the extracts included the determination of total phenolic content and antioxidant capacity by FRAP and ABTS assays. Total phenolic content for red GSE presented higher values (2.77-18.3I $\mathrm{mg}$ gallic acid $\mathrm{g}^{-1}$ sample) when compared with those for white GSE (2.28-5.25 $\mathrm{mg}$ gallic acid $\mathrm{g}^{-1}$ sample) (Fig. 4A). In accordance with this, red GSE showed higher reducing power and free radical scavenging capacity than white GSE. FRAP results ranged between 28.9 and $337.0 \mu \mathrm{mol}$ TEAC $\mathrm{g}^{-1}$ extract in the case of red GSE and between 29.2 and I0I.3 $\mu$ mol TEAC $g^{-1}$ extract for white GSE (Fig. 4B). The ABTS results varied from 18.8 to $101.2 \mu \mathrm{mol}$ TEAC $\mathrm{g}^{-1}$ extract for red GSE and from 22.5 to $53.7 \mu \mathrm{mol}$ TEAC $\mathrm{g}^{-1}$ extract for white GSE (Fig. 4C).

\section{DISCUSSION}

MGO is one of the promoters of AGE formation as measured in glycation assays. Furthermore, it has been demonstrated that MGO is formed in the early stage of glycation from the degradation of glucose, Schiff base adduct, 3-deoxyglucosone or fructosamine. ${ }^{6}$ The present study examined the MGO trapping activity of methanolic extracts from different red and white grape skins obtained from winemaking by-products of different cultivars. The aim was to evaluate whether MGO trapping activity could explain the overall antiglycative properties of the extracts, previously demonstrated. ${ }^{16,17}$ For this purpose, different concentrations of GSE were incubated in the presence of MGO under physiological conditions $(\mathrm{pH} 7.4$, $37^{\circ} \mathrm{C}$ ) for $168 \mathrm{~h}$. The concentration required for each of the samples to trap $50 \%$ of the MGO present in the reaction medium was estimated. As mentioned above, a kinetic study was performed in order to evaluate the trapping efficiency for each extract throughout the $168 \mathrm{~h}$ experiment at $24 \mathrm{~h}$ intervals. In this experiment, the time necessary for each of the samples to trap $50 \%$ of the MGO present in the reaction medium was calculated.

According to the result obtained, both red and white GSE exhibited promising MGO scavenging capability, as shown in Fig. I. This may explain the mechanism involved in the ability of the extracts to inhibit AGEs formation, as previously reported. ${ }^{16,17}$ Some differences were found between red and white GSE, which could be due to both the varying chemical composition of the extracts and their behavior in the model reaction systems.

It is well known that grapes are rich sources of polyphenolic compounds such as anthocyanins, flavanols, flavonols and proanthocyanidins, all of which possess antioxidant and antiglycation properties. ${ }^{14,27}$ It can therefore be expected that similar beneficial effects can be attained from winemaking by-products. $16,17,28$ There is evidence that polyphenols play a major role in direct MGO trapping capacity. ${ }^{29,30}$ In agreement with this, $I C_{50}$ trapping capacity for red GSE was found to be significantly correlated with their total phenolic content $\left(r^{2}=-0.8835, P=0.0196\right)$ (Fig. $\left.4 A\right)$ and consequently with FRAP values $\left(r^{2}=-0.8444, P=0.0344\right)$ (Fig. 4B) and $A B T S$ values $\left(r^{2}=-0.8655, P=0.0259\right)$ (Fig. 4C).

However, despite the fact that the direct MGO trapping results for both red and white GSE were very close, the aforementioned correlation was not observed in white extracts (Fig. 4A, B, C), which suggests that in these samples other compounds were involved in MGO trapping. This could imply that components from red GSE tend to inhibit AGE generation by directly quenching reactive carbonyl compounds. Anthocyanins are a major class of flavonoids in red grapes, and their ability to trap MGO has been 
demonstrated in blackcurrant berries,3I this inhibiting efficiency being associated with delphinidin 3rutinoside and cyanidin 3-rutinoside. However, in the case of white GSE, mechanisms other than dicarbonyl trapping may be involved in their antiglycative properties. ${ }^{16,17}$ Similar results have been reported by other authors, who did not find any correlation between phenolic content or antioxidant capacity and anti-AGE activity. ${ }^{23,32}$ The reason could be that polyphenols are not the sole antiglycative agents present in the extracts and therefore other compounds might be contributing to their overall anti-AGE activity. ${ }^{33}$ In addition, polyphenols could have either synergistic or antagonistic effects on other constituents in the reaction medium, thus affecting carbonyl trapping capacity. ${ }^{34}$

\section{CONCLUSIONS}

The in vitro MGO trapping capacity of six red and seven white GSE obtained from winemaking by-products was investigated. Methanolic GSE could inhibit the formation of AGEs by direct trapping of MGO under physiological conditions. The high efficiency of the extracts was proven by the short time required to trap $50 \%$ of the dicarbonyl compound. In conclusion, GSE possess good carbonyl trapping abilities and could be the source of a natural dietary antiglycation agent. It also opens up the opportunity for their utilization as a possible nutraceutical ingredient during fortification studies. However, further research is required to understand the mechanism involved in the inhibitory activities of red and white grape skin samples.

\section{ACKNOWLEDGMENTS}

PSC Sri Harsha would like to thank the University of Milan for providing additional funding through the ERASMUS student network program. This work was partly funded by projects CSIC-201370E027, AGER (project number 2010-2222) and S2013/ABI-3028-AVANSECAL.

\section{REFERENCES}

I Russ W and Pittroff RM, Utilizing waste products from the food production and processing industries. Crit Rev Food Sci Nutr 44:57-62 (2004).

2 Seeram NP, Lee R, Scheuller HS and Heber D, Blackberry, black raspberry, blueberry, cranberry, red raspberry, and strawberry extracts inhibit growth and stimulate apoptosis of human cancer cells in vitro. J Agric Food Chem 54:9329-9339 (2006).

3 McDougall GJ, Ross HA, Ikeji M and Stewart D, Berry extracts exert different antiproliferative effects against cervical and colon cancer cells grown in vitro. J Agric Food Chem 56:3016-3023 (2008).

4 Gordillo G, Fang H, Khanna S, Phillips G and Sen CK, Oral administration of blueberry inhibits angiogenic tumor growth and enhances survival of mice with endothelial cell neoplasm. Antioxid Redox Signal I I:47-58 (2009).

5 Anhe FF, Desjardins Y, Pilon G, Dudonné S, Genovese MI, Lajolo FM et al., Polyphenols and type 2 diabetes: a prospective review. Pharma Nutrition I:I05-II4 (20I3).

6 Wang $Y$ and Chi-Tang H, Flavour chemistry of methylglyoxal and glyoxal. Chem Soc Rev 4I:4I40-4I49 (20I2).

7 Singh R, Barden A, Mori T and Beilin L, Advanced glycation end-products: a review. Diabetologia 44:I29146 (200I).

8 Odani H, Shinzato T, Matsumoto Y, Usami J and Maeda K, Increase in three alpha, beta-dicarbonyl compound levels in human uremic plasma: specific in vivo determination of intermediates in advanced Maillard reaction. Biochem Biophys Res Commun 256:89-93 (1999).

9 Vander Jagt DL, Hassebrook RK, Hunsaker LA, Crown WM and Royer RE, Metabolism of the 2oxoaldehyde methylglyoxal by aldose reductase and by glyoxalase-l: roles for glutathione in both enzymes and implications for diabetic complications. Chem Biol Interact I30-132:549-562 (200I). 
10 Yim HS, Kang SO, Hah YC, Chock PB and Yim MB, Free radicals generated during the glycation reaction of amino acids by methylglyoxal: a model study of protein-cross-linked free radicals. J Biol Chem 270:2822828233 (1995).

II Rahbar S and Figarola JL, Novel inhibitors of advanced glycation endproducts. Arch Biochem Biophys 419:63-79 (2003).

12 Matsuda H, Wang T, Managi $\mathrm{H}$ and Yoshikawa M, Structural requirements of flavonoids for inhibition of protein glycation and radical scavenging activities. BioorgMed Chem II:53 I7-5323 (2003).

13 Dorsey PG and Greenspan P, Inhibition of nonenzymatic protein glycation by pomegranate and other fruit juices. J Med Food 17:447-454 (2014).

14 Wang W, Yagic $Y$ and Buran TJ, Phytochemicals fromberries and grapes inhibited the formation of advanced glycation end-products by scavenging reactive carbonyls. Food Res Int 44:2666-2673 (201 I).

$15 \mathrm{Wu} \mathrm{CH}$ and Yen GC, Inhibitory effect of naturally occurring flavonoids on the formation of advanced glycation endproducts. J Agric Food Chem 53:3167-3173 (2005).

16 Sri Harsha PSC, Gardana C, Simonetti P, Spigno G and Lavelli V, Characterization of phenolics, in vitro reducing capacity and anti-glycation activity of red grape skins recovered from winemaking by-products. Bioresour Technol 140:263-268 (2013).

17 Sri Harsha PSC, Lavelli V and Scarafoni A, Protective ability of phenolics from white grape vinification byproducts against structural damage of bovine serum albumin induced by glycation. Food Chem 156:220-226 (2014).

I8 Saraswat M, Reddy PY, Muthenna P and Reddy GB, Prevention of nonenzymatic glycation of proteins by dietary agents: prospects for alleviating diabetic complications. Br J Nutr I0 I:I7|4-172I (2009).

19 Dearlove RP, Greenspan P, Hastle DK, Swanson RB and Hargrove JL, Inhibition of protein glycation by extracts of culinary herbs and spices. J Med Food II:275-28I (2008).

20 Ho SC, Pei S, Wu S, Lin SM and Tang YL, Comparison of antiglycation capacities of several herbal infusions with that of green tea. Food Chem 122:768-774 (2010).

2I Reddy VP and Beyaz A, Inhibitors of the Maillard reaction and AGE breakers as therapeutics for multiple diseases. Drug Discov Today I I:646-654 (2006).

22 Peng X, Cheng KW, Ma J, Chen B, Ho CT, Lo C et al., Cinnamon bark proanthocyanidins as reactive carbonyl scavengers to prevent the formation of advanced glycation endproducts. J Agric Food Chem 56:1907-191I (2008).

23 Mesías M, Navarro M, Gökmen V and Morales FJ, Antiglycative effect of fruit and vegetable seed extracts: inhibition of AGE formation and carbonyl trapping abilities. J Sci Food Agric 93:2037-2044 (2013).

24 Singleton VL, Orthofer R and Lamuela-Raventós RM, Analysis of total phenols and other oxidation substrates and antioxidants by means of Folin-Ciocalteu reagent. Meth Enzymol 299:152-178 (1999).

25 Morales FJ, Martin S, Açar OC, Arribas-Lorenzo G and Gökmen V, Antioxidant activity of cookies and its relationship with heat-processing contaminants: a risk/benefit approach. Eur Food Res Technol 228:345-354 (2009).

26 Delgado-Andrade $\mathrm{C}$ and Morales FJ, Unraveling the contribution of melanoidins to the antioxidant activity of coffee brews. J Agric Food Chem 53:1403-1407 (2005).

27 De la Cruz AA, Hilbert G, Mengin V, Rivière C, Ollat N, Vitrac $C$ et al., Anthocyanin phytochemical profiles and anti-oxidant activities of Vitis candicans and Vitis doaniana. Phytochem Anal 24:446-452 (2013).

28 Jariyapamornkoon N, Yibchok-anun S and Adisakwattana S, Inhibition of advanced glycation end products by red grape skin extract and its antioxidant activity. BMC Complement Altern Med I3:I7I (2013).

29 Lo CY, Li S, Tan D, Pan MH, Sang S and Ho CT, Trapping reactions of reactive carbonyl species with tea polyphenols in simulated physiological conditions. Mol Nutr Food Res 50:I I I8-I I 28 (2006). 
30 Shao X, Bai N, He K, Ho CT, Yang CS and Sang S, Apple polyphenols, phloretin and phloridzin: new trapping agents of reactive dicarbonyl species. Chem Res Toxicol 21:2042-2050 (2008).

3I Chen XY, Huang IM, Hwang LS, Ho CT, Li S and Lo CY, Anthocyanins in blackcurrant effectively prevent the formation of advanced glycation end products by trapping methylglyoxal. J Funct Foods 8:259-268 (2014).

32 Povichit N, Phrutivorapongkul A, Suttajit M, Chaiyasut CC and Leelapornpisid P, Phenolic content and in vitro inhibitory effects on oxidation and protein glycation of some Thai medicinal plants. Pak J Pharm Sci 23: 403-408 (2010).

33 Sun Z, Peng X, Liu J, Fan KW and Wang M, Inhibitory effects of microalgal extracts on the formation of advanced glycation endproducts (AGEs). Food Chem I20:26I-267 (20I0).

34 Rice-Evans CA, Miller NJ and Paganga G, Structure-antioxidant activity relationships of flavonoids and phenolic acids. Free Radic Biol Med 20:933-956 (1996). 


\section{FIGURES}

Figure I. Dose-dependent results for MGO trapping capacity experiment at $168 \mathrm{~h}$ of methanolic grape skin extracts (GSE): (A) red GSE; (B) white GSE. Samples with similar trends were grouped and represented with the same trend line (red GSE: R-GI: FR ; R-G2:CR, DO , R-G3: BA ; R-G4:GR ; R-G5: NR ; white GSE: WGI: CH, MO ; W-G2:MT, AR,NA ; W-G3: ER, RI ). PM solution (0.I $\left.\mathrm{mgmL}^{-1}\right)$ presented an MGO trapping capacity of $99 \%$ (not shown). For clarity only the higher error bars are represented.
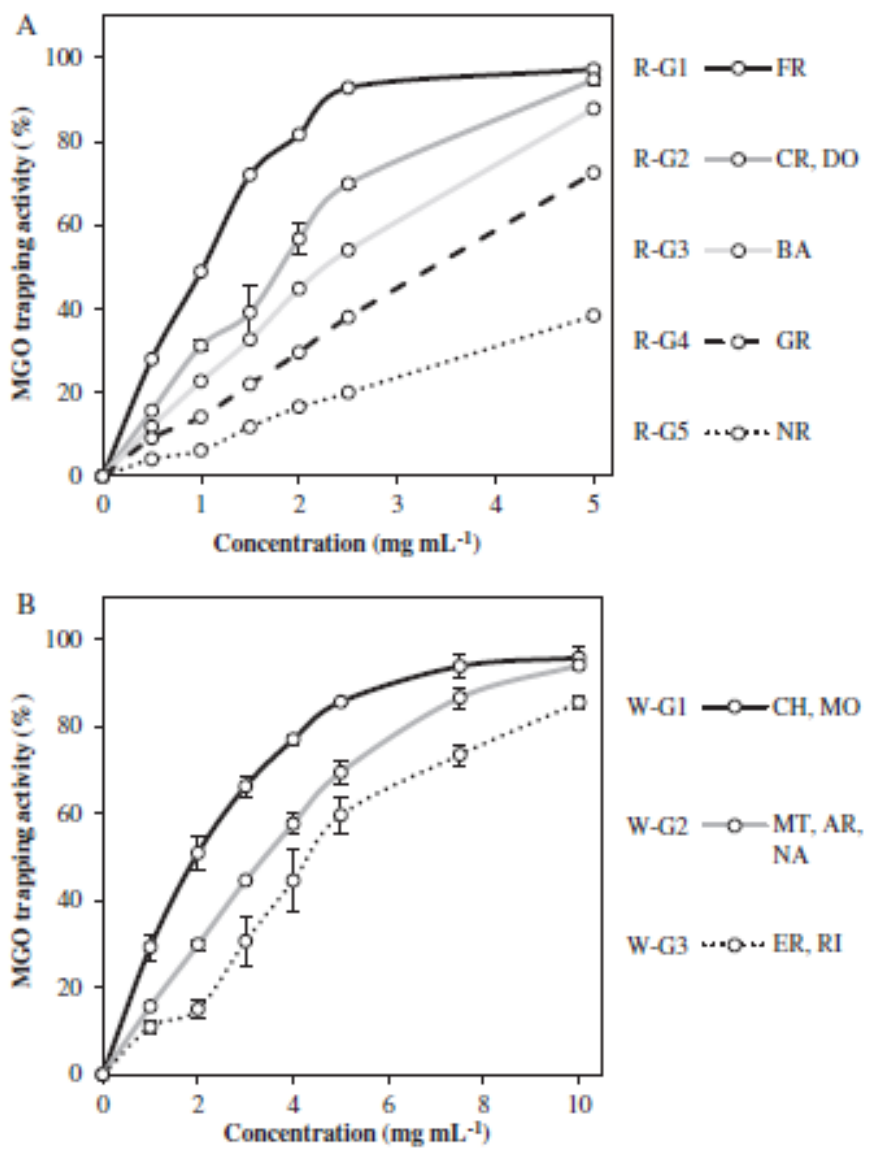
Figure 2. Box-plot representation and estimated $\mathrm{IC}_{50}\left(\mathrm{mgmL}^{-1}\right)$ for $\mathrm{MGO}$ trapping capacity experiment at $168 \mathrm{~h}$ of methanolic grape skin extracts (GSE): Red GSE, ; white GSE, . Results are expressed as mean \pm SD; $\mathrm{n}=4$. Different letters mean significant differences $(P<0.05)$. PM presented an IC50 of $0.005 \mathrm{mgmL}^{-1}$. Asterisk indicates outlier data.

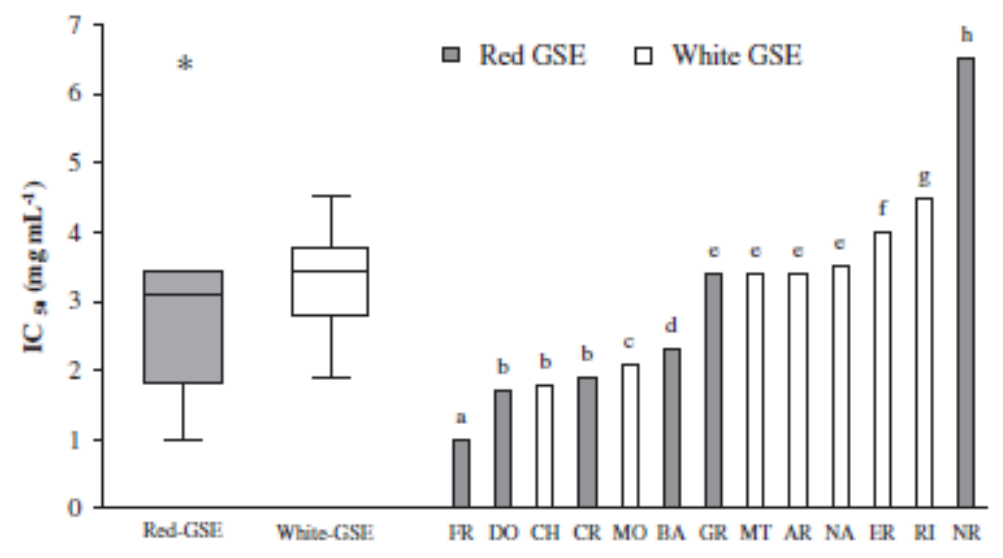


Figure 3. Kinetic study of direct MGO trapping efficiency of methanolic grape skin extracts (GSE) for 168 h: (A) red GSE at $2.5 \mathrm{mgmL}^{-1}$; (B) white GSE at $5 \mathrm{mgmL}^{-1}$. Samples were grouped and represented as in Fig. I. Results are expressed as mean $\pm S D ; n=4$. PM solution (final concentration $0.1 \mathrm{mgmL}^{-1}$ ) represented an MGO trapping capacity of $98 \%$.
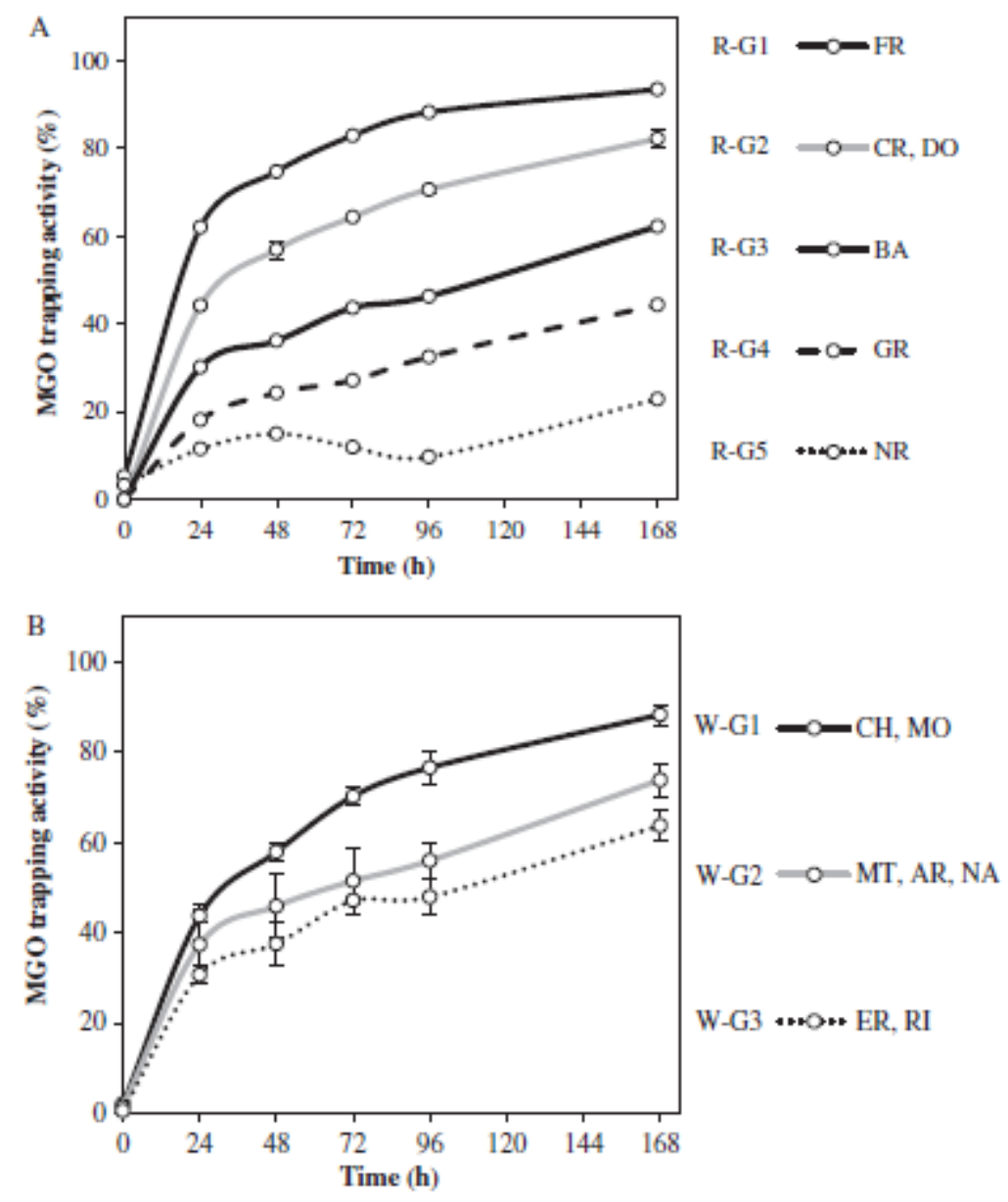
Figure 4. Correlation between MGO trapping capacity, total phenolic content and antioxidant capacity. Correlation between $\mathrm{IC}_{50}\left(\mathrm{mgmL}^{-1}\right)$ for MGO trapping capacity and (A) total phenolic content (TPC), (B) results from FRAP assays and $(C)$ results from $A B T S$ assay for red GSE, ; and white GSE, . GAE, gallic acid equivalent; TEAC, Trolox equivalent antioxidant capacity.
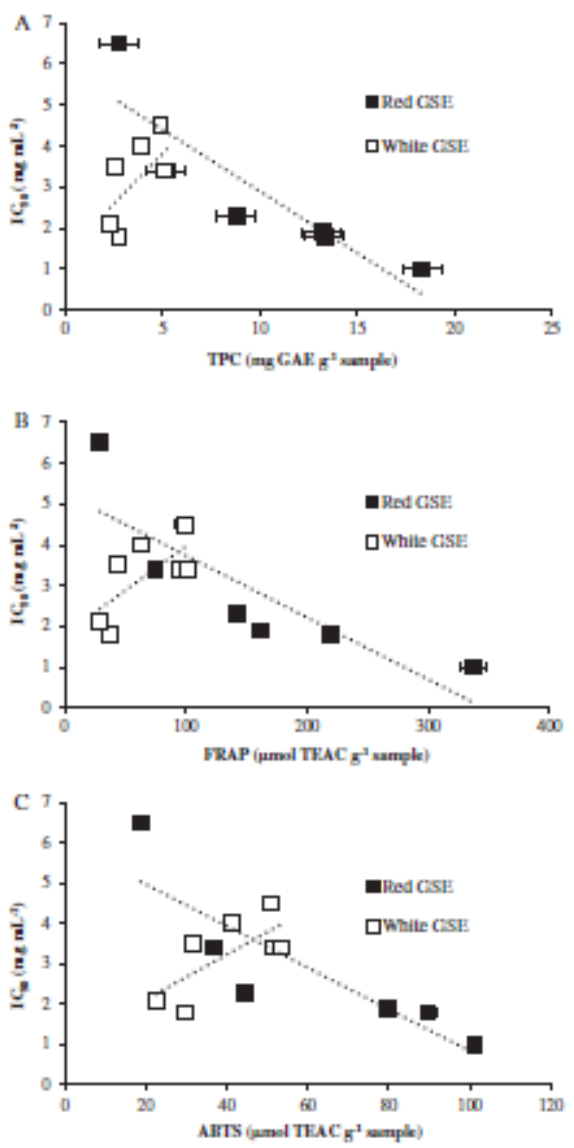